\title{
Clinical Impact of Blood Culture Results in Acutely III Hospitalized Adult Patients With Cystic Fibrosis
}

\author{
Robert J. Vender ${ }^{\mathrm{a}}$, Robert L. Vender ${ }^{\mathrm{b}, \mathrm{c}}$
}

\begin{abstract}
Background: Blood cultures are obtained clinically to confirm site and source of acute infection as well as to guide effective antibiotic therapies. Patients with cystic fibrosis (CF) are at risk for blood stream infection (BSI) as identified from positive blood culture results.
\end{abstract}

Methods: A retrospective chart review was performed of 190 adult CF patients from January 1, 2001 through December 1, 2015. All positive blood culture results were identified as to clinical relevance and source of BSI.

Results: There were a total of 3,053 blood cultures. One hundred fifty-one positive blood cultures were considered pathogenic and clinically significant. Venous access device-related BSI was identified in 31 evaluable patients and 106 blood cultures. Nineteen patients and 45 positive blood cultures were attributable to organ-specific sources.

Conclusion: Two patterns of BSI were identified: 1) venous access device infections without causal mortality and 2) organ-specific site infections with associated $26 \%$ mortality.

Keywords: Blood stream infection; Positive blood culture results

\section{Introduction}

Blood cultures for microbiological analyses are commonly obtained to assist in providing a confirmatory diagnosis of acute infections in a number of clinical areas and diseases. In addition, many institutions require surveillance blood cultures prior to initiation of any antibiotic therapies [1]. When blood

Manuscript accepted for publication September 27, 2016

aPenn State College of Medicine, 500 University Drive, Hershey, PA 17033, USA

${ }^{b}$ Department of Medicine, Penn State Milton S. Hershey Medical Center, 500 University Drive, Hershey, PA 17033, USA

${ }^{\mathrm{c} C o r r e s p o n d i n g ~ A u t h o r: ~ R o b e r t ~ L . ~ V e n d e r, ~ D e p a r t m e n t ~ o f ~ M e d i c i n e, ~ P e n n ~}$ State Milton S. Hershey Medical Center, 500 University Drive, Hershey, PA 17033, USA. Email: rvender@psu.edu

doi: http://dx.doi.org/10.14740/jocmr2764w culture results are positive, this information represents invaluable data in assisting in antibiotic selection, defining site and potential source of primary infectious origin, and prognosis. General guidelines and prediction rules have been proposed to assist in identifying patients with relatively high probability of positive blood cultures which would then represent true positives and confirmation of blood stream infection (BSI) [2]. However, the vast majority of blood culture results are negative with reported rates of true positive culture results ranging between approximately $4 \%$ and $10 \%$, depending upon specific clinical indication. Perhaps even more damaging from a clinical perspective is when the results represent contamination and thus would confuse the clinical management with these false positive results not indicative of factual BSI [3-6].

Cystic fibrosis (CF) represents a disease manifested by both acute and chronic sustained lung infections. Most adult $\mathrm{CF}$ patients have received multiple courses of antibiotic therapy, either inhaled as a component of chronic lung heath maintenance, or acutely as inpatient care for acute infectious exacerbations [7]. In addition, adult CF patients frequently have indwelling vascular access devices (foreign bodies) for prolonged durations, which creates another source for both local and BSI [8-10]. In this review, we report the analysis of over 3,000 blood culture results in 150 total adult CF patients over a 15 -year period of time from a single institution to define the frequency, clinical impact, and pathogens (both bacterial and fungal) for this disease-specific BSI.

\section{Methods}

This study was approved including waiver of informed consent by the Human Subjects Protection Office and Institutional Review Board of the Penn State Milton S. Hershey Medical Center (HMC). Adult CF patients (18 years of age or older) were identified by the continuous log of active and deceased patients kept daily by the CF Foundation accredited adult program director and coordinator at HMC. Retrospective chart review was performed over the investigational period of January 1, 2001 through December 1, 2015. All blood cultures obtained during this period of time were reviewed and the results were recorded. Afterwards the medical record was reviewed to specifically identify the relevance, impact, site source, therapies, and patient outcome related to each positive blood culture result and associated BSI. 
Table 1. Frequency of Bacterial and Fungal Related Venous Access Device Infections and Associated Bacteremia and Fungemia in Adult Patients ( $n=31$ ) With Cystic Fibrosis (2001 - 2015)

\begin{tabular}{llll}
\hline & Patients & Events & $\begin{array}{l}\text { Positive blood } \\
\text { cultures }\end{array}$ \\
\hline Bacterial: catheter infections & 4 & 6 & 14 \\
Fungal: catheter infections & 1 & 1 & 2 \\
Bacterial: TIVAP infections & 13 & 23 & 48 \\
Fungal: TIVAP infections & 13 & 17 & 42 \\
Total & 31 & 47 & 106 \\
\hline
\end{tabular}

TIVAP: totally implantable venous access ports. Event: single temporal identified infectious complication-noting multiple patients had more than one event. Three positive (+) blood cultures were polymicrobial.

\section{Results}

During the time period of January 1, 2001 through December 1, 2015, there were 190 adult CF patients registered at the $\mathrm{HMC}$ adult $\mathrm{CF}$ program, including 53 deaths over that time period. Forty patients did not have blood cultures obtained, which yielded 150 total evaluable adult CF patients with recorded blood culture results. There was a total of 3,053 blood cultures obtained with a mean number per patient equal to 20.5 \pm 22.4 (SD) and a range of $1-122$ cultures per patient. There were a total of $175(6 \%)$ positive blood cultures with an identi- fied potentially pathogenic microbial agent. Upon review of the medical record, two positive blood cultures were of undefined clinical significance or etiology (methicillin-sensitive Staphylococcus aureus, viridans Streptococcus), and 22 were presumed contaminants and not treated as to clinical significance (2: methicillin-resistant Staphylococcus aureus and 20: Staphylococcus epidermidis and other coagulase-negative Staphylococcus, Corynebacterium, Oerskovia, Micrococcus species). The remaining 151 (5\%) positive blood cultures were considered pathogenic and clinically significant. Four positive blood cultures were polymicrobial (Pseudomonas aeruginosa + methicillin-resistant Staphylococcus aureus; Pseudomonas aeruginosa + Enterobacter aerogenes; vancomycin-resistant Enterococcus faecalis (VRE) + Candida glabrata; Enterococcus faecalis (not VRE) + Candida albicans).

Venous access device-related BSIs included sources of catheter-related infections and totally implantable venous access ports (TIVAPs). Catheter-related sources included percutaneously inserted central venous catheters, peripherally inserted central venous catheters (PICCs), and dialysis catheters. Thirty-one (21\%) of evaluable patients developed a venous access device-related BSI which accounted for 106 of $151(70 \%)$ positive blood cultures. Sixteen (11\%) of these positive blood cultures were related to venous catheters and the majority 90 $(60 \%)$ were related to TIVAP. Results for both bacterial and fungal-related venous access device infections with associated BSI are presented in Tables 1 and 2.

Organ-specific infectious sources clinically determined to contribute to the positive blood cultures and disseminated

Table 2. Venous Access Device Related Blood Stream Infections in Adult Patients $(n=31)$ With Cystic Fibrosis (2001 - 2015): Frequency (numerical value relates to total number of positive blood cultures) and Species of Pathogenic Infecting Microbial Agent

\begin{tabular}{ll}
\hline Bacterial & Fungal \\
\hline Catheter-related blood stream infections & \\
$\begin{array}{l}\text { Enterococcus faecalis (not VRE): } 6 \\
\text { Coagulase-negative Staphylococcus: } 4\end{array}$ & Candida glabrata: 2 \\
Methicillin-resistant Staphylococcus aureus: 1 & \\
Vancomycin-resistant Enterococcus faecalis (VRE): 3 & \\
Totally implantable venous access port (TIVAP) blood stream infections & \\
Methicillin-resistant Staphylococcus aureus: 18 & Candida albicans: 22 \\
Coagulase-negative Staphylococcus: 10 & Candida glabrata: 12 \\
Vancomycin-resistant Enterococcus faecalis (VRE): 1 & Candida tropicalis: 6 \\
Enterococcus faecalis (not VRE): 4 & Candida parapsilosis: 2
\end{tabular}

Methicillin-sensitive Staphylococcus aureus: 4

Serratia marcescens: 3

Enterobacter aerogenes: 3

Pseudomonas aeruginosa: 2

Enterobacter cloacae: 1

Flavobacterium species: 1

Klebsiella oxytoca: 1 
Table 3. Lung-Specific Related Blood Stream Infections in Adult Patients With Cystic Fibrosis ( $n=10)$ (2001 - 2015): Frequency (Numerical Value Relates to Total Number Positive Blood Cultures) and Species of Pathogenic Infecting Microbial Agent

\begin{tabular}{ll}
\hline Methicillin-resistant Staphylococcus aureus & 6 \\
Methicillin-resistant Staphylococcus aureus & 1 \\
Methicillin-resistant Staphylococcus aureus + Pseudomonas aeruginosa & 1 \\
Hemophilus influenza & 1 \\
Stenotrophomonas maltophilia & 1 \\
& \\
Burkholderia cepacia complex & $4^{*}$ \\
Burkholderia cepacia complex & $1^{*}$ \\
Burkholderia cepacia complex (+ cavitary lung disease) & 2 \\
Burkholderia cepacia complex (+ cavitary lung disease) & $1^{*}$ \\
Burkholderia cepacia complex (+ cavitary lung disease) & 1 \\
\hline${ }^{*}$ Cause of death. &
\end{tabular}

BSIs occurred in 19 (13\%) evaluable adult CF patients and 45 $(30 \%)$ of all positive blood cultures. Despite the relatively low frequency of blood stream dissemination from primary lung infection in CF patients reported in the literature, 10 episodes of BSI in this review derived from a lung source (noting five episodes involved Burkholderia cepacia complex (BCC)). In addition, nine BSIs resulted from non-lung etiologies. As opposed to the venous access device-related BSIs, where none of the infections were thought to be causally related to mortality, BSIs from organ-specific infections were thought to be the cause of death in five patients. Lung-related sources of BSIs are listed in Table 3 and non-lung sources in Table 4.

\section{Discussion}

Although lacking any defined abnormalities or deficiencies in adaptive humoral or cellular immunity and acknowledging the well-established defect in innate host defense affecting the lungs of individuals with $\mathrm{CF}$, adult patients with $\mathrm{CF}$ clearly manifest numerous host defense limitations that place them at risk for infection [11]. These potential risk factors include the unique milieu of the CF lung that allows for the sustained proliferation of highly pathogenic bacteria, multiple invasive procedures and foreign bodies that remain in situ indefinitely with resultant frequent manipulations, malnutrition and CF-related diabetes, propensity for renal calculi and bowel obstruction often requiring invasive surgical procedures, and near constant exposure to antibiotics [12]. This report highlights the impact of many such parameters upon the morbidity and mortality of adult CF patients.

From a clinical perspective, these positive blood culture results and associated BSIs can be divided into two categories, primary infections related to nosocomial contamination of venous access devices and secondary BSIs resulting from localized organ-specific infections with secondary hematogenous dissemination.

The majority of BSIs [70\%] resulted from invasive indwelling venous catheters, foreign bodies, and TIVAP. These BSIs were caused by a wide variety of both bacterial and fun-

Table 4. Non-Lung Organ-Specific Related Blood Stream Infections in Adult Patients With Cystic Fibrosis ( $\mathrm{n}=9$ ) (2001 - 2015): Frequency (Numerical Value Relates to Total Number Positive Blood Cultures) and Species of Pathogenic Infecting Microbial Agent

Endocarditis (tricuspid valve)

Pyelonephritis/kidney abscess

Pyelonephritis

Urinary tract infection with sepsis

PEG tube/abdominal wall cellulitis

PEG tube/abdominal wall cellulitis

Wound dehiscence/bowel evisceration/peritonitis

Spontaneous bacterial peritonitis/cirrhosis

$\begin{array}{ll}\text { Methicillin-resistant Staphylococcus aureus } & 3 \\ \text { Candida glabrata } & 4 * \\ \text { Alcaligenes xylosoxidans } & 3 \\ \text { Enterococcus faecalis (not VRE) and methicillin-resistant Staphylococcus aureus } & 2,2 \\ \text { Methicillin-resistant Staphylococcus aureus } & 3 \\ \text { Methicillin-resistant Staphylococcus aureus } & 1 \\ \text { Candida glabrata and Candida parapsilosis } & 3^{*}, 2^{*} \\ \text { Klebsiella pneumoniae and methicillin-resistant Staphylococcus aureus } & 1,1\end{array}$

Salmonella species: $S$. muenster serogroup $1+$ S. alachla serogroup 0

${ }^{*}$ Cause of death. PEG: percutaneous endoscopic gastrostomy. 
gal infecting pathogens $[13,14]$. Although not specifically assessed in this retrospective chart review, it must be anticipated with high probability that these primary nosocomial infections would have significant negative health outcomes both for patients (symptoms, additional procedures, hospitalizations, prolonged lengths of stay, additional antibiotics), heath care providers, and resources (costs and resource utilization). Despite being a potential source of burden and disease to adult $\mathrm{CF}$ patients, these venous access device-related infections were not causally linked to mortality.

In contrast, the majority of mortality from BSIs resulted from catastrophic organ infections associated with tissue destruction and dissemination into the blood stream systemically. Although lacking histological or autopsy evidence in all cases, retrospective review of the medical records for each patient clearly documented clinical, laboratory, and radiographic supporting evidence for these invasive infections. For non-lungrelated organ-generated BSIs, we particularly highlight the occurrences of methicillin-resistant Staphylococcus aureus bacterial endocarditis and pyelonephritis/kidney abscess resultant from Candida glabrata infection, the latter being fatal. In relation to lung-generated BSIs, our data again reflect current knowledge in emphasizing the invasive pathogenicity of $\mathrm{BCC}$. In this report, $\mathrm{BCC}$ caused extensive cavitary lung disease in three patients and was directly responsible for death in three patients. This is in contrast to the single identified case of Pseudomonas aeruginosa lung dissemination which was not fatal. It is worth noting that Pseudomonas aeruginosa is the most common infectious agent in the lungs of patients with CF based upon 2014 CF Foundation Patient Registry data approximating $66 \%$, while $\mathrm{BCC}$ lung infection was reported in only $3.4 \%[15]$.

In summary, based upon this single institution study over a 15-year period, we have identified a high prevalence of disseminated BSIs based upon positive blood culture results. Theoretically, 31 evaluable patients (21\%), 47 events, and 106 (70\%) of the positive blood cultures due to venous catheter or TIVAP-related infections were preventable. The invasive nature of BCC lung infection and propensity of BCC bacteria to invade the blood stream leading to the development of sepsis from "Cepacia syndrome" which is associated with a high mortality was again evident $[12,16,17]$.

\section{Conflicts of Interest}

None.

\section{Funding Support}

None.

\section{References}

1. Coburn B, Morris AM, Tomlinson G, Detsky AS. Does this adult patient with suspected bacteremia require blood cultures? JAMA. 2012;308(5):502-511.

2. Shapiro NI, Wolfe RE, Wright SB, Moore R, Bates DW. Who needs a blood culture? A prospectively derived and validated prediction rule. J Emerg Med. 2008;35(3):255264.

3. Weinstein MP, Towns ML, Quartey SM, Mirrett S, Reimer LG, Parmigiani G, Reller LB. The clinical significance of positive blood cultures in the 1990s: a prospective comprehensive evaluation of the microbiology, epidemiology, and outcome of bacteremia and fungemia in adults. Clin Infect Dis. 1997;24(4):584-602.

4. Towns ML, Jarvis WR, Hsueh PR. Guidelines on blood cultures. J Microbiol Immunol Infect. 2010;43(4):347349.

5. Roque P, Oliver B, Anderson L, Mulrow M, Drachman $\mathrm{D}$, Stapczynski S, LoVecchio F. Inpatient utilization of blood cultures drawn in an urban ED. Am J Emerg Med. 2012;30(1):110-114.

6. Ratzinger F, Dedeyan M, Rammerstorfer M, Perkmann T, Burgmann H, Makristathis A, Dorffner G, et al. A risk prediction model for screening bacteremic patients: a cross sectional study. PLoS One. 2014;9(9):e106765.

7. Gibson RL, Burns JL, Ramsey BW. Pathophysiology and management of pulmonary infections in cystic fibrosis. Am J Respir Crit Care Med. 2003;168(8):918-951.

8. Aitken ML, Tonelli MR. Complications of indwelling catheters in cystic fibrosis: a 10-year review. Chest. 2000;118(6):1598-1602.

9. Kariyawasam HH, Pepper JR, Hodson ME, Geddes DM. Experience of totally implantable venous access devices (TIV ADs) in adults with cystic fibrosis over a 13-year period. Respir Med. 2000;94(12):1161-1165.

10. Burdon J, Conway SP, Murchan P, Lansdown M, Kester RC. Five years' experience of PAS Port intravenous access system in adult cystic fibrosis. Eur Respir J. 1998;12(1):212-216.

11. Ratjen F, Doring G. Cystic fibrosis. Lancet. 2003;361(9358):681-689.

12. O'Sullivan BP, Freedman SD. Cystic fibrosis. Lancet. 2009;373(9678):1891-1904.

13. Gaber KA, Shenton L, Oades P, Colville A, Sheldon CD. Fungal septicaemia in patients with cystic fibrosis associated with totally implantable venous access device system. J Cyst Fibros. 2007;6(5):317-319.

14. Parienti JJ, Mongardon N, Megarbane B, Mira JP, Kalfon P, Gros A, Marque S, et al. Intravascular Complications of Central Venous Catheterization by Insertion Site. N Engl J Med. 2015;373(13):1220-1229.

15. Cystic Fibrosis Foundation. 2014 Cystic Fibrosis Foundation Patient Registry, Bethesda, MD. 2014.

16. Frangolias DD, Mahenthiralingam E, Rae S, Raboud JM, Davidson AG, Wittmann R, Wilcox PG. Burkholderia cepacia in cystic fibrosis. Variable disease course. Am J Respir Crit Care Med. 1999;160(5 Pt 1):1572-1577.

17. De Boeck K, Malfroot A, Van Schil L, Lebecque P, Knoop C, Govan JR, Doherty C, et al. Epidemiology of Burkholderia cepacia complex colonisation in cystic fibrosis patients. Eur Respir J. 2004;23(6):851-856. 